\section{Gestörter Schlaf durch Allergie}

\author{
Erholsamer Schlaf ist entscheidend für das physische und psy- \\ chische Wohlbefinden - und gerade bei Allergikern überdurch- \\ schnittlich häufig nicht gegeben.
}

\begin{abstract}
A llergologen der italienischen Universität Genua haben eine praxisrelevante Übersicht erstellt zu Schlafstörungen bei Allergikern, die mit Fragebögen einfach ermittelt werden können. Die wichtigsten Resultate sind nachfolgend zusammengefasst.

Bei der Rhinitis allergica, vor allem bei der obstruktiven Variante, kommt es häufig zu Schnarchen und chronischer Tagesmüdigkeit, wobei auch die Erholungsfähigkeit eingeschränkt ist. Dies tritt auch bei der saisonalen Rhinitis auf. Je schwerer die Rhinitissymptome, desto
\end{abstract}

gravierender sind auch die Schlafprobleme.

Patienten mit Asthma bronchiale haben ebenfalls häufig Schlafstörungen, da sich die Atemnot meist nächtlich verstärkt und mit Husten und Giemen einhergeht. Der Index für Lebensqualität sinkt ab. In einer Telefonbefragung von 2.800 Asthmatikern klagten 30\% über Schlafstörungen.

Beim atopischen Ekzem sind ebenfalls Schlafstörungen und Tagesmüdigkeit belegt. Tritt das Ekzem zusammen mit Asthma bronchiale und Rhinitis all- ergica auf, finden sich im Vergleich zu Gesunden deutlich mehr Schlafstörungen mit den bereits genannten Folgeerscheinungen.

Bei der chronischen Urtikaria schließlich führt der nachts sich verstärkende Juckreiz oft zu Einschlaf- und Durchschlafstörungen.

\section{Fazit}

Schlafstörungen sind bei Allergikern überdurchschnittlich häufig und meist dem Bereich der Dyssomnien zuzuordnen. Sie sollten in Diagnostik und Therapie berücksichtigt werden. Dabei ist insbesondere die sedierende Wirkung der Antihistaminika zu berücksichtigen.

$$
\text { Dr. Dieter Bruchhausen, Wuppertal }
$$

\section{Literatur}

Baiardini I, Braido F, Caglia S, Canonica GW. Sleep disturbances in allergic diseases. Allergy 2006; 61: 1259-67

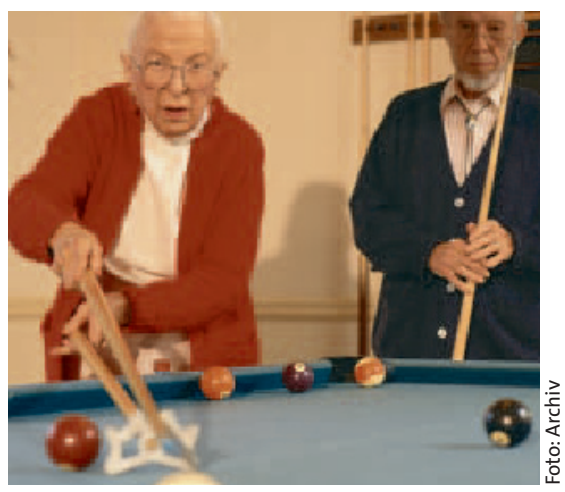

Die über 65-Jährigen bilden in vielen Industriestaaten die am stärksten wachsende Bevölkerungsgruppe.

zurechtkommen. Sie wenden ihre Medikamente regelmäßig an und haben trotz schlechterer Lungenfunktion eine höhere Lebensqualität. Problematisch ist dagegen offenbar die Kommunikation mit den Medizinern, hier wären Gesprächskontakte mit erfahrenen Fachärzten wünschenswert.

\section{Dr. Dieter Bruchhausen, Wuppertal}

\section{Literatur}

Slavin RG, Haselkorn T, Lee JH, Zheng B, Deniz Y, Wenzel SE. Asthma in older adults: observations from the epidemiology and natural history of asthma: outcomes and treatment regimens (TENOR) study. Ann Allergy Asthma Immunol 2006; 96: 406-14 